\section{Regards sur l'économie allemande}

Bulletin économique du CIRAC

$110 \mid 2013$

Varia

\title{
Les enjeux du débat allemand sur le travail de qualité
}

Isabelle Bourgeois

\section{(2) OpenEdition}

Journals

Édition électronique

URL : http://journals.openedition.org/rea/4588

DOI : $10.4000 /$ rea. 4588

ISSN : 1965-0787

Éditeur

CIRAC

Édition imprimée

Date de publication : 15 octobre 2013

Pagination : 5-18

ISSN : 1156-8992

\section{Référence électronique}

Isabelle Bourgeois, "Les enjeux du débat allemand sur le travail de qualité », Regards sur l'économie

allemande [En ligne], 110 | octobre 2013, mis en ligne le 01 octobre 2015, consulté le 05 mai 2019.

URL : http://journals.openedition.org/rea/4588 ; DOI : 10.4000/rea.4588 


\section{Les enjeux du débat allemand sur le travail de qualité}

\author{
Isabelle Bourgeois
}

A en croire les médias français qui s'emparent avec un plaisir non dissimulé des arguments de la campagne électorale de la gauche allemande et relaient ainsi dans l'opinion française la mobilisation européenne contre l'austérité de la Confédération européenne des syndicats (CES) et d'autres organisations, il n'y aurait outre-Rhin que précarité et pauvreté - l'envers de la compétitivité économique. Plus de 7 millions d'Allemands seraient forcés de travailler pour des salaires de misère. Avec des patrons par nature exploiteurs, des salariés victimes d'un système " ultra-libéral ", comment les Allemands pourraient-ils être épanouis et heureux ? Dans ces conditions, la qualité de vie au travail, le travail de qualité, voire le travail décent ne peuvent être que de vains mots en Allemagne.

Pourtant, à considérer les études dignes de foi et réalisées en Allemagne ou au sein de l'Union européenne - des travaux scientifiques jusqu'aux analyses du mouvement syndical -, les Allemands se distinguent de leurs voisins européens sur plusieurs aspects, et principalement celui-ci : ils se disent heureux au travail. Et si parfois la sécurité de l'emploi leur cause souci, l'épanouissement personnel dans un environnement propice à la confiance leur importe bien plus que le montant de leur salaire. Leur priorité n'est pas l'introduction d'un salaire minimum légal, qu'il soit de 8,50 € l'heure comme le voudrait la Confédération syndicale $D G B$ ou de $10 €$ comme le prône Die Linke. Ce qui compte à leurs yeux, c'est le respect et la reconnaissance que leur vaut leur travail. Sur ce point, ils sont au fond très proches des Français pour qui, si on se réfère à la dernière enquête de la Sofres sur la qualité de vie au travail (juin 2013), celle-ci se résume à trois notions : respect, reconnaissance et épanouissement.

Alors, qu'en est-il de la qualité du travail en Allemagne? Ou, pour le formuler comme la Confédération DGB : qu'est-ce qui caractérise le "bon travail » (gute Arbeit) ? A considérer de près les termes du débat mené outre-Rhin, on s'aperçoit que, contrairement à ce qu'on veut faire croire en France, la question d'un 'meilleur' travail est loin de constituer la préoccupation première des salariés allemands; elle n'est et n'était qu'un argument de campagne politique et syndicale centré sur des thèmes ciblés (salaire, sécurité de l'emploi). Ensuite, si les médias français (et allemands) s'emparent si volontiers de cette thématique, c'est que la notion même de travail/emploi de qualité (ou de qualité de vie au travail) s'y prête idéalement, tant elle est floue, les comparaisons internationales reposant sur des statistiques partielles ou peu comparables entre elles. Enfin, ce flou sert l'instrumentalisation transfrontières de la problématique pauvreté/précarité dans un agenda setting anticapitaliste et anti-mondialisation dont la finalité est l'adoption " d'amortisseurs sociaux 》 européens en compensation des « contraintes » liées à l'UEM.

\section{« Bon travail » - arguments politiques, construction et finalité}

"Nous avons tous le droit à avoir un bon travail ». C'est ainsi que s'ouvre, sur le site de la Confédération syndicale DGB, la rubrique de ses positions dans le cadre des élections au Bundestag (www.dgb.de/btw2013/gute-arbeit). Qui ne répondrait pas par «oui » si une telle question lui était posée au cours d'un sondage d'opinion ? Cette formulation est très/trop générale - intentionnellement -, comme dans tout programme destiné à susciter une large adhésion.

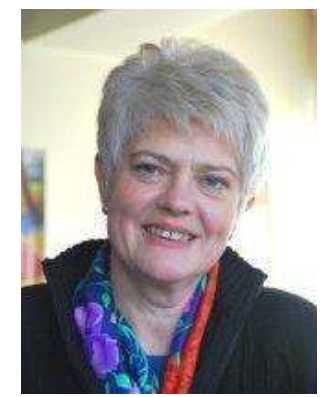

Isabelle Bourgeois, chargée de recherche au CIRAC et rédactrice en chef de Regards sur l'économie allemande

Les positions du DGB dans la campagne électorale 
«Bon travail » : un concept fédérateur car indéfini
Après avoir posé sa première revendication - des syndicats puissants -, la Confédération DGB décline ce qu'elle entend par « bon travail ». Sous l'intitulé "Nous devons adopter une nouvelle organisation du travail ", il est précisé : "Nous avons besoin de plus d'emplois à durée indéterminée, soumis à protection sociale, et avec une rémunération suffisante pour vivre - en lieu et place de bas salaires et d'emplois précaires. Car la valeur du travail ne doit pas être dépréciée plus longtemps ». Vient ensuite, sous le titre "Nous méritons un travail meilleur ", en quelque sorte l'exposé des motifs: "Nous avons besoin de plus d'emplois, mais pas à n'importe quel prix, c'est la mauvaise approche. Nous avons besoin de pouvoir concilier vies familiale et professionnelle, de meilleures conditions de travail et d'une protection plus efficace de la santé face au burnout, au stress et au mobbing. Car le travail ne doit pas rendre malade ". Voilà donc rassemblées en vrac, sous l'appellation « bon travail » les approches les plus diverses : rémunération (élevée), durée du contrat (CDI), couverture sociale ou critères de qualité de vie au travail (stress...). Cette présentation aussi est consensuelle. De plus, elle est conforme à l'image renvoyée actuellement par les médias (allemands) qui se focalisent sur le stress au travail et le burnout, deux thématiques fortes dans l'espace public car correspondant aux préoccupations politiques nationales et européennes.

\section{Quelques chiffres sur l'emploi atypique et les bas salaires}

\section{Emploi atypique}

Selon Destatis, en 2012, on dénombrait 7,89 millions de salariés occupant un emploi atypique, soit $21,8 \%$ des actifs occupés. Si cette part a considérablement progressé depuis 1991, où elle n'était encore que de $12,8 \%$, elle a atteint le maximum en 2007 $(22,6 \%)$ et baisse depuis. Dans le même temps, la part des salariés occupant un emploi " classique » (normal ou régulier) est en constante hausse depuis 2006 ; elle atteignait 24,2 millions en 2012 (communiqué du 28-08-2013).

Destatis définit l'emploi atypique par contraste avec l'emploi dit régulier (CDI, plus de 21 heures hebdomadaires, couverture sociale). L'emploi atypique regroupe donc : temps partiels de 20 heures par semaine ou moins, " petits boulots », CDD et emplois intérimaires. Les actifs considérés sont âgés de 15 à 64 ans, ne poursuivent ni scolarité/études ni apprentissage, ne sont pas militaires ni actifs dans le service civil (www.destatis.de/DE/Meta/AbisZ/AtypischeBe schaeftigung.html).

Dans cette fiche de définition consacrée à cette catégorie, Destatis explique que, contrairement à l'emploi régulier, les emplois atypiques ne permettent pas d'assurer entièrement ou à eux seuls la subsistance de celui qui les occupe (et de ses proches). Et précise : " mais il n'est pas possible de les assimiler à des emplois précaires. L'emploi précaire se caractérise en effet par un risque de pauvreté supérieur du/des personnes qui l'occupe(nt), qui dépend en outre de sa biographie professionnelle comme du type de ménage dans lequel il vit. Les formes d'emploi atypique décrites ici peuvent parfaitement avoir été choisies parce qu'elles permettent par exemple de mieux concilier, dans un cas donné, intérêts professionnels et autres intérêts personnels ॥.

\section{Bas salaires}

Pour les statisticiens, un bas salaire est un salaire inférieur aux deux tiers du salaire médian. Rien ne permet d'en tirer une quelconque corrélation avec le seuil de (risque de) pauvreté dont le calcul prend en considération le salaire brut, mais aussi les revenus de transferts et l'unité de consommation. La base de calcul pour les données sur les niveaux des salaires est exclusivement celle des salaires bruts horaires. "Pour un célibataire, même un bas salaire peut suffire pour vivre, ce qui n'est pas le cas d'un salarié qui doit assurer la subsistance d'une famille nombreuse grâce à son seul salaire » (Rhein, 2013).

Selon Destatis, le seuil des bas salaires s'établit en Allemagne à 10,36 € (bruts) par heure en 2010 (dernières données disponibles), ce qui porte la part de ce segment à $20,6 \%$ dans la structure des salaires. Sont pris en considération dans ce calcul les salariés de 15 à 64 ans, sans les apprentis et les actifs en pré-retraite, et occupés dans des entreprises d'au moins 10 salariés, quel que soit le secteur d'activité - hormis l'agriculture et l'administration publique (source : Niedriglohn und Beschäftigung. Begleitmaterial zur Pressekonferenz am 10. 09. 2012 in Berlin). Ces données alimentent en partie l'Enquête sur la structure des salaires (ESS) menée tous les quatre ans par Eurostat. L'ESS 2012 parvient aux taux suivants : $17 \%$ de bas salaires dans I'UE 27 et $22,2 \%$ en Allemagne (6,1\% en France ; communiqué Eurostat $\left.n^{\circ} 189 / 2012\right)$.

Il existe une multitude d'autres sources de données sur le seuil des bas salaires, qui se distinguent toutes par des modes de calcul différents. Selon les calculs de l'institut IAB, qui se base sur les données EU-SILC et les révise quelque peu, ce seuil est de $9,54 €$ en 2010. La population concernée est celle des 20-64 ans sauf les apprentis. Ces calculs diffèrent de ceux d'EU-SILC dans la mesure où ils reposent sur une durée régulière du temps de travail d'au moins sept heures hebdomadaires, ce qui inclut les mini-jobs. Par ailleurs, contrairement à la plupart des statistiques sur l'écart des salaires qui ne prennent en compte que l'emploi principal, celles de I'IAB considèrent également les boulots d'appoint ; le temps de travail et le salaire de l'emploi principal sont alors additionnés. Selon ces calculs, l'ensemble du secteur des bas salaires représente $24,1 \%$ du total de l'emploi en Allemagne (IAB-Kurzbericht, $\mathrm{n}^{\circ}$ 15/2013).

Autrement dit, le montant horaire du seuil des bas salaires et, partant, la part de ce secteur dans la structure des salaires varient beaucoup selon les sources considérées. Les données publiées sont donc à manier avec prudence.

Un 'coupable' : la politique « néo-libérale »
Dans un discours tenu le 29 mai à Berlin et consacré à cette nouvelle organisation du travail, Michael Sommer, président du DGB, explicite l'enjeu : "Nous avons besoin d'une nouvelle organisation du travail parce que le marché du travail allemand est profondément divisé après trois décennies de dérégulation 
néo-libérale et de démantèlement des droits des salariés ". Si on fait abstraction du sociolecte syndical, un tel constat ne peut que faire l'unanimité. Effectivement, le marché du travail allemand n'est plus le même aujourd'hui que dans les années 1980 : le travail féminin est en constante hausse, il est hautement qualifié, les biographies professionnelles ne sont plus aussi linéaires, débutant souvent par un ou plusieurs CDD, et les « petits boulots » qui bénéficient d'un régime fiscal spécifique depuis leur création à la fin des années 1960 se sont multipliés (voir Spitznagel, 2009). Qu'on résume cette évolution sous le terme de mutation du travail ou de précarisation (imputée, dans le contexte de la campagne électorale, aux réformes « Hartz » ; voir REA 108/2013), le constat est le même, mais pas l'appréciation qu'on lui porte.

Quand on revendique plus de « bon travail », on part de deux présupposés. On pose d'abord comme évidente la généralisation d'un « mauvais travail » contre laquelle il s'agit de lutter. Et on crée de la sorte une thématique suffisamment globale pour réunir une large adhésion et une forte mobilisation; et de fait, ce thème s'est hissé en tête de l'agenda politique durant la campagne aux élections du Bundestag (en France plus qu'en Allemagne) pour diffuser une vision misérabiliste des conditions de travail outre-Rhin appelant une réponse urgente. Si dans son discours, Michael Sommer se garde de l'exprimer aussi explicitement, le communiqué de presse le résumant est, lui, on ne peut plus clair: "L'intérim, les mini-jobs et les contrats d'entreprise sont synonymes de mauvais travail ». Voilà donc désignés les 'coupables' et nommé l'enjeu principal de la lutte pour une nouvelle organisation du travail : re-réguler l'emploi atypique. M. Sommer poursuit ainsi : comme l'Allemagne est l'Etat européen qui a «le plus gros secteur des bas salaires », il faut y remédier en introduisant un salaire minimum légal généralisé de 8,50 € afin de "prendre en considération les besoins des salariés ॥. Voilà le deuxième présupposé : l'emploi de qualité se mesure au montant du salaire perçu, ce montant doit être fixé par le législateur, il doit être le même pour tous, quelle que soit l'activité exercée ou la branche du salarié, et il se définit par rapport au socialement souhaitable.

Ce qui est étonnant dans ce programme, ce n'est pas la revendication d'un salaire présenté comme décent ou suffisant pour en vivre ; elle appartient au répertoire classique de tout syndicat. Ce qui l'est en revanche beaucoup plus, c'est de voir la Confédération DGB revendiquer un salaire minimum légal alors que, en Allemagne, les syndicats et leurs homologues patronaux sont seuls habilités par la Constitution (Loi fondamentale, art. $9 \S 3$ ) à fixer salaires et horaires de travail au niveau de leur branche (autonomie tarifaire). Les conventions ainsi conclues ont en quelque sorte force de loi pour la profession. Le législateur n'a aucune compétence en la matière, sauf en cas de défaillance des partenaires sociaux (voir par exemple REA 88/2008). II dispose alors de trois possibilités : (1) le recours à la Loi sur les conditions de travail minimales (Mindestarbeitsbedingungengesetz, MiArbG), une loi de 1952 qui n'a jamais été appliquée avant sa révision en 2009 ; (2) la procédure d'extension (Allgemeinverbindlichkeitserklärung) prévue par la Loi sur les conventions tarifaires, qui s'applique en cas "d'urgence sociale », mais qui est peu utilisée ; (3) le recours à la Loi sur les travailleurs détachés (Arbeitnehmer-Entsendegesetz, AEntG) de 1996 dont l'objectif officiel est la lutte contre le travail illégal mais à laquelle il a été recouru à plusieurs reprises pour écarter le risque de « dumping salarial » et introduire un SMIC légal dans 12 branches/métiers à ce jour (du BTP à la coiffure en passant par les services de sécurité).

Et de fait, la première revendication de la plateforme électorale du DGB porte bien sur des syndicats puissants : "L'autonomie tarifaire doit être préservée, et la cogestion doit être développée. Avec des syndicats forts en nombre d'adhérents... ". Le mouvement syndical plaide en fait depuis longtemps pour un recours systématique et élargi à la procédure d'extension qui permet de concilier régulation de branche et régulation par la loi, puisque le législateur se contente de 'régulariser' en les généralisant des accords conclus entre les partenaires
Une seule solution : un SMIC légal

Appel à l'Etat vs autonomie tarifaire? 
Le DGB prône un SMIC légal depuis 2006

La représentativité syndicale est en recul depuis l'Unité

Montée en puissance de la concurrence inter-syndicale sociaux au niveau d'une branche (voir Bispinck/Schulten, 2009). D'un côté donc, le DGB en appelle au législateur, officialisant de la sorte la défaillance des syndicats, et d'un autre côté, il continue de se réclamer de sa responsabilité en tant que partenaire social. Comment expliquer cette contradiction?

Ces positions sont loin d'être nouvelles. II y a quatre ans déjà, dans un contexte comparable - élections au Bundestag, suivies quelques mois plus tard par les élections européennes, et sur fond de négociations du volet social des politiques communautaires -, les mêmes types d'arguments étaient propagés (voir REA 86/2008). En réalité, l'idée d'un SMIC légal avait timidement germé plus tôt encore : en 2003, mais elle avait été rejetée au cours des négociations sur les réformes « Hartz ». Depuis, elle resurgit à intervalles réguliers, et le DGB en a fait ouvertement son cheval de bataille à partir de 2006.

Si la revendication revient en force aujourd'hui, c'est essentiellement pour deux raisons. La première est structurelle. Depuis le milieu des années 1990, le modèle de la convention tarifaire de branche au fondement du partenariat social allemand subit une lente et constante érosion (voir Lattard, 2005). Sous l'effet de la globalisation, des mutations du travail et de la flexibilisation croissante du tissu productif, la référence de branche tend à s'effriter, entraînant le recul de la représentativité syndicale (et patronale). Le taux de syndicalisation est tombé de $36 \%$ en 1991 à quelque $20 \%$ aujourd'hui, et le nombre de membres du DGB est aujourd'hui inférieur à ce qu'il était avant l'Unité, passant de 7,94 millions en 1990 à un pic de 11,80 en 1991, avant de décroître continûment pour tomber à 6,15 millions en 2012. Quant au taux de couverture des salariés par les conventions de branche, il est lui aussi en constant recul : en 2012, seulement $53 \%$ des salariés à l'ouest (48\% dans le privé) et $36 \%$ à l'est $(29 \%$ dans le privé) étaient couverts (WSI-Mitteilungen, $n^{\circ} 4 / 2013$ ), la différence estouest s'expliquant par la politique démesurée de hausse salariale dans l'exRDA menée immédiatement après l'unification, qui a accéléré la désaffection patronale et donc l'appartenance des entreprises à une organisation patronale. En 1996 encore, ces taux étaient de respectivement $70 \%$ et $56 \%$.

Certains secteurs, surtout les services qui se sont rapidement développés depuis le milieu des années 1990 ou sont nés de la vague de la privatisation d'anciens services publics, n'ont jamais pu être totalement organisés, faute d'organisations patronales ou parce que les nouvelles entreprises créées ne s'affilient pas à une organisation patronale, ou encore du fait de la multiplication des externalisations. Les moins organisés sont de loin les services cantonnés au marché domestique ; ceux qui sont exposés à la concurrence mondiale sont, au contraire, bien organisés. C'est dès lors dans ces services (gastronomie, services à la personne, etc.) que se trouvent la plupart des emplois atypiques, notamment les mini-jobs (Kalina/Weinkopf, 2013). A la faiblesse institutionnelle du syndicalisme s'ajoute depuis quelques années la concurrence inter-syndicale. Elle oppose d'un côté Ver.di et IG Metall sur le découpage des branches et donc leur domaine d'influence respectif, principalement dans le secteur des services aux entreprises industrielles (voir REA 86/2008). Cette concurrence oppose de l'autre les syndicats du DGB (modèle du syndicat unitaire de branche) et les nouveaux syndicats catégoriels comme Cockpit (pilotes), le Marburger Bund (médecins) ou GDL (conducteurs de locomotives) par exemple. Cette évolution a mené à une situation paradoxale où certaines catégories professionnelles, parce qu'elles ont un fort pouvoir de nuisance du fait de leur positionnement stratégique dans la chaîne de création de valeur, se livrent à une surenchère salariale, alors qu'à l'autre extrême, nombre de salariés voient leurs salaires stagner, faute de représentation syndicale. Enfin, en 2010, le Tribunal fédéral du Travail a levé le principe de syndicat unique (un syndicat par branche et par entreprise), estimant que peuvent coexister plusieurs conventions signées par plusieurs syndicats. C'est aussi à ces évolutions que fait allusion le constat de division du marché du travail, voire de sa fracture, et la nécessité de le réorganiser, formulé par $\mathrm{M}$. Sommer dans le discours cité. 
L'autre raison qui sous-tend la revendication d'un SMIC légal (comme critère de « bon travail ») est conjoncturelle. Dans la succession de crises depuis 1993 et l'effort de reconquête de la compétitivité économique, le syndicalisme allemand a exercé pleinement sa responsabilité macro-économique en accompagnant la modernisation du tissu productif. Mais en se rangeant à la raison économique, il a quelque peu perdu de vue sa mission de "justice sociale ". Depuis la crise de restructuration mondiale de l'industrie qui avait plongé l'économie allemande dans la récession et menacé son industrie (1993), les partenaires sociaux s'étaient imposés une 'règle d'or' pour les conventions collectives : la hausse des salaires conventionnels doit toujours être légèrement inférieure à la hausse de la productivité. Cette politique a permis aux entreprises industrielles de se restructurer, de redevenir compétitives, de croître, d'investir et de créer des emplois. Cette modération salariale raisonnée et l'accompagnement par les syndicats dans la gestion de crise (voir Schröder, 2013) sont l'une des raisons pour lesquelles l'économie allemande a pu surmonter les crises, y compris celle de 2008/09, particulièrement sévère, et rester compétitive. Or c'est justement cette capacité du tissu productif à résister, la stabilité de l'emploi dont elle s'accompagne, de même que la croissance qu'elle entretient, du moins en tendance, qui incite à l'optimisme et nourrit aujourd'hui les convoitises, ravivant la tentation de procéder à la redistribution des richesses. Cette tentation est aussi entretenue de l'extérieur, et notamment en provenance de France où la modération allemande est souvent désignée comme la cause du différentiel de compétitivité au sein de la zone Euro et du décrochage de l'économie française.

Plus fondamentales encore sont les répercussions sur l'identité syndicale de la crise de la finance mondiale et de la crise de la dette souveraine au sein de la zone Euro. Elles ont forcé un changement de paradigme dans l'approche syndicale de la politique pour l'emploi, reposant sur le constat : " il faut s'attendre à ce que sous le vieux slogan 'L'essentiel, c'est d'avoir un travail' la réduction de la qualité des conditions de vie au travail soit érigée en système pour se décharger de la gestion de crise et en faire supporter le coût aux salariés" (Pickshaus, 2009). La crise est dès lors perçue comme une opportunité pour un renouveau syndical. Comme le mouvement syndical ne parvient plus à relever seul le défi de la " division » du marché du travail, " une nouvelle dialectique entre Etat et partenaires sociaux s'impose... Dans ce partage du travail, il devrait revenir dans un premier temps à l'Etat de veiller à un minimum de sécurité et d'orientation - par exemple au moyen d'un SMIC légal généralisé. Cela permettrait, dans un deuxième temps, aux partenaires sociaux de mener une politique de la raison éclairée afin d'améliorer la qualité des relations entre les partenaires sociaux et celle du marché du travail » (Schroeder, 2013). Vue sous cette angle, la revendication d'un SMIC légal généralisé comme garant d'un « bon travail » n'entre pas en contradiction avec l'autonomie tarifaire ; elle vise à rétablir les forces du mouvement syndical sur une nouvelle base et à "restabiliser le système de la convention de branche » (Bispinck/Schulten, 2009).

La période actuelle aussi crée un contexte porteur pour un changement de paradigme. D'une part, la nouvelle Stratégie Europe 2020 de l'Union européenne, qui prolonge la Stratégie de Lisbonne adoptée en 2000 (son horizon était 2010, son slogan : "croissance et emploi »), poursuit deux objectifs : le développement d'une économie dynamique (compétitivité) et le renforcement de la cohésion sociale. Dans cette "croissance intelligente, durable et inclusive " à atteindre d'ici 2020, le volet social est résumé par la formule : " plus d'emplois de meilleure qualité ". Cette préoccupation de la qualité se décline en l'occurrence sous la forme d'objectifs de hausse de l'emploi féminin, de l'emploi des seniors et des actifs faiblement qualifiés. Elle se conjugue à d'autres, plus anciennes, comme cet accord visant à assurer une protection minimale contre le stress au travail, conclu entre les partenaires sociaux européens multisectoriels en 2004. Or dans l'évaluation qu'elle a fait en 2011 de la mise en œuvre de cet accord, la Commission regrette que "les résultats obtenus ... en Allemagne... n'ont pas
Remise en question de la doctrine de la modération salariale

La crise dans I'UE, une opportunité pour un renouveau syndical

Europe 2020 : "plus d'emplois de meilleure qualité » 
Qualité du travail, facteur clé de compétitivité

Crise de 2008 : thérapie de choc pour un changement de paradigme

"Bon travail »= un concept destiné à la re-mobilisation répondu aux attentes » (IP/11/223). Voilà un autre élément permettant de nourrir la revendication d'un meilleur travail, d'autant que, depuis 2007, le DGB fait effectuer tous les ans une enquête représentative sur le "bon travail »: le $D G B-I n d e x$ Gute Arbeit, et que le stress comme la sécurité au travail y sont régulièrement thématisés. La Confédération a également publié la même année un "Manuel du Bon Travail » (Handbuch Gute Arbeit) destiné aux conseils d'entreprise et qui les sensibilise à la prévention. Les médias relayent abondamment ces questions qui permettent d'associer dramatisation et touche humaine, surtout sous l'angle du « management by stress ».

Le thème de la qualité du travail est loin d'être une préoccupation exclusivement syndicale. En 2002 par exemple avait vu le jour en Allemagne une Initiative nouvelle qualité du travail (Initiative Neue Qualität der Arbeit) réunissant Bund, Länder, fédérations professionnelles, syndicats, entreprises, l'Agence fédérale pour l'emploi, caisses d'assurance sociale et fondations. L'objectif partagé de ce forum d'échange de best practices en matière de management, d'équité des chances, de santé et de savoirs/compétences, est de promouvoir " une meilleure qualité du travail comme facteur clé de la capacité d'innover et de la compétitivité du site Allemagne » (www.inqa.de). C'est dans ce contexte notamment qu'avait été développé un baromètre pour mesurer le stress au travail (Stressbarometer) qui avait débouché sur le manuel afférent du DGB. II s'agit ici d'améliorer moins le travail considéré globalement que, très concrètement, la gouvernance des entreprises et la gestion des ressources humaines qui sont en Allemagne un des facteurs clés de l'innovation process, produit, mais aussi sociétale (capacité à s'adapter au changement). Et dans cette approche holistique du concept d'innovation, le mouvement syndical est en pointe. En 2004, par exemple, le DGB avait publié une plaquette s'intitulant : " En quoi l'innovation concerne-t-elle les syndicats ? "(voir REA 90/2009). Elle développait un axe central de son programme rénové : la hausse des qualifications, et plaidait pour une participation active des partenaires sociaux dans la reconquête de la compétitivité allemande dans une économie globalisée.

Depuis la crise de la finance mondiale et ses répercussions au sein de la zone Euro, le concept d'innovation a pris une dimension supplémentaire dans la politique syndicale. Elle vise le renouveau du syndicalisme en tant qu'acteur de la polis et propage dans ce cadre la vision d'un nouveau modèle de société en rupture avec le dogme "tayloriste et toyotiste ". Selon les chercheurs proches du milieu syndical, la crise de la finance mondiale appelle en effet une " thérapie de choc " pour rompre avec " une pensée dominée par les marché financiers, le concept de shareholder value qui en découle avec sa vision court-termiste, et qui s'est traduite par le délaissement systématique des ressources humaines " (Kalmbach/Schumann, 2008). Faisons abstraction du fait que la culture de la shareholder value ne s'est jamais généralisée dans une Allemagne où les entreprises sont à $95 \%$ familiales et partagent une culture de la stakeholder value et d'orientation sur le long terme. La question qui se pose pour le syndicalisme est dès lors : comment opérer un "changement de paradigme » susceptible de déboucher à terme sur un "new deal » tel qu'il avait suivi la crise de 1929 ? Pour l'Europe, la crise de 2008/09 est idéale : "la résolution de la crise financière pourrait créer l'impulsion nécessaire pour développer des stratégies et des solutions permettant d'aborder l'ensemble des questions à résoudre avec une nouvelle qualité d'approche. Non plus donc sous celle d'une communauté de dérégulation, mais dans la perspective d'une meilleure réalisation transnationale d'une politique obéissant à la raison sociétale » (ibid.).

Dans ce contexte, la revendication d'une meilleure qualité du travail, partagée par le DGB et la gauche politique allemande, prend une nouvelle dimension : celle de la revitalisation de la puissance syndicale. Pour ce faire, les « syndicats ont renouvelé le concept de l'humanisation du monde du travail, créé naguère pour le monde du travail de l'ère fordiste, lui conférant la valeur stratégique de bon travail pour guider leur action dans l'entreprise, mais aussi leur agenda set- 
ting dans l'espace public » (Brinkmann/Nachtwey, 2010). II s'agit bien d'une autre vision du rôle du syndicalisme dans l'organisation de la société. Le thème du « bon travail » a pour unique fonction d'entretenir la mobilisation - en Allemagne et au-delà. Elle suit plusieurs objectifs et se décline sous divers aspects.

"Bon travail - un thème réservé aux beaux jours ou un élément dans une stratégie offensive pour surmonter la crise " (Pickshaus, 2009). Ce thème fédérateur est un outil servant la propagation d'une nouvelle identité : la représentativité syndicale traversant une "crise grave ", le syndicalisme doit se refonder en tant que " puissance d'organisation » (Dörre, 2008). Les penseurs-stratèges proches du milieu syndical identifient deux axes de renouveau : une " restabilisation par la base " et une "d'en haut » (Bispinck/Schulten, 2009). II s'agit donc de réinvestir l'entreprise comme lieu de négociation, via les conseils d'entreprise, pour remédier à la fonte de la représentativité au niveau de la branche. Pour ce faire, il faut amener les salariés à s'organiser et à adhérer à leur syndicat de branche. Les syndicats ont donc développé des stratégies ciblées d'identification des secteurs à organiser, des entreprises à viser - de préférence internationalisées ou fortement réputées, afin de créer un fort écho médiatique - et sont passés maîtres dans l'art de l'agenda setting. Le dernier exemple en date est la campagne nationale, européenne et mondiale de campaigning menée au début de l'année 2013 par Ver.di contre Amazon (voir REA 108/2013). A cette reconquête de la base s'ajoute le défi de parvenir, 'en haut', à une "r restabilisation politique du système allemand de la convention de branche " (Bispinck/ Schulten, 2009). L'outil en est l'introduction d'un SMIC légal qui présente l'avantage de créer, dans toutes les branches non organisées, " une base réglementaire pour la mise en place, ensuite, de relations tarifaires » (ibid.). Pour y parvenir, le " principal levier politique " est le recours à la procédure d'extension " comme c'est le cas dans la plupart des autres Etats de l'UE ».

C'est dans cette optique que le DGB avait créé, il y a quelques années, un portail internet dédié à ce SMIC (www.mindestlohn.de); mais c'était essentiellement sous l'impulsion de Ver.di. Car le camp syndical reste divisé sur ce point. Deux cultures s'opposent, incarnées par les deux principaux concurrents : celle, traditionnelle, du syndicat unitaire de branche (IG Metall, mais aussi IG BCE), et la fonction tribunicienne que s'est donnée le conglomérat Ver.di (" plus Greenpeace que puissance gréviste », Frank Bsirske). Pour Ver.di, le recours à la loi est la condition sine qua non de sa propre légitimation; pour IG-Metall, elle reste un pis-aller. "La réorganisation du marché du travail ne peut se faire seulement via le recours à la loi. Il appartient aux syndicats de créer, en organisant mieux leurs adhérents, les conditions permettant de stabiliser l'autonomie de la politique tarifaire et de la reconquérir dans les zones qui y sont actuellement soustraites ». C'est ainsi que Gerhard Bosch, directeur de l'institut de recherche $I A Q$ auprès de l'université de Duisburg, résume le constat qu'il a établi dans le cadre d'un rapport d'expertise réalisé pour le compte d'IG Metall et consacré au " travail précaire et à la réorganisation du marché du travail » (IAQ-Standpunkt, 2/2012). Ce rapport est l'un des documents cherchant à étayer scientifiquement les programmes des syndicats dans la campagne électorale et leur revendication d'un SMIC légal généralisé.

Parallèlement, le thème du « bon travail » poursuit aussi un objectif politique et idéologique transnational. Ver.di en est la force la plus active. L'enjeu inclut la reconquête du pouvoir d'organisation et le dépasse : il s'agit de reconquérir le « pouvoir institutionnel ». Le moyen est une approche de la mobilisation importée des Etats-Unis, et qui est en train de profondément modifier la culture syndicale allemande : l'organizing. L'élément déterminant en est "le passage d'un modèle de la représentativité syndicale fondé sur le modèle d'un prestataire de services cherchant à retenir des membres généralement passifs par des prestations qualifiées, vers un modèle de type organizing, qui mise sur la mobilisation des adhérents, modifie le mode de travail des organisations à la base et crée de nouvelles structures organisationnelles participatives » (Dörre, 2013). Si les
Reconquérir la base et repositionner le syndicalisme

Deux cultures syndicales

Bâtir un autre modèle de société 
Mobiliser en réseaux

Associer les scientifiques

Construire une Europe sociale outils de l'action stratégique sont pluriels, ils reposent sur quelques éléments clés. Pour organiser les intérêts faiblement représentés, en l'occurrence les salariés occupant un emploi précaire et, dans ce groupe, les femmes, il convient de placer dans l'opinion des thèmes porteurs et fédérateurs. "La campagne sur le salaire minimum légal portée par plusieurs syndicats membres du DGB prend dans ce contexte une fonction politico-symbolique » (ibid.).

Encore faut-il construire la thématique « bon travail » dans l'opinion en mobilisant "partis politiques, syndicats, mouvements citoyens » (Kalmbach/Schumann, 2008). Dans la campagne aux élections du Bundestag de 2013, l'alliance des syndicats avec Die Linke (un parti issu de la fusion entre les communistes de l'ancien parti-Etat de la RDA et la mouvance trotskiste rassemblant d'anciens syndicalistes) et d'autre part, un parti social-démocrate aux prises avec une profonde crise identitaire et tenté de se repositionner sur sa gauche, cette construction s'est visiblement révélée efficace. Mais il convient aussi de mobiliser les 'mieux organisés' que sont la mouvance anti-mondialiste, les ONG et, plus généralement, tous les réseaux sociaux et mouvements protestataires (Dörre, 2013). Ver.di est précurseur en la matière : dès le début du siècle, il a forgé des alliances stratégiques hors du champ syndical, notamment avec ATTAC, ainsi lors des grandes manifestations européennes contre la Directive Services en 2005 (voir REA 71/2005) et l'instrumentalisation de cette Directive, insérée dans la revendication d'une Europe sociale, lors du référendum français sur le Traité constitutionnel. Et en Allemagne, depuis 2008, année de la libéralisation des services postaux immédiatement suivie de l'adoption d'un salaire minimum sectoriel légal (voir REA 85/2008), le principal axe politique de Ver.di se résume par ce slogan : "Pauvre malgré un travail » (Arm trotz Arbeit).

Pour que les campagnes portent leurs fruits et soient crédibles, "la qualité de la coopération entre syndicalistes et scientifiques est déterminante " et doit se conjuguer à "des structures coopératives adéquates entre scientifiques, journalistes et professionnels de la communication " (Dörre, 2008). Un exemple en est l'institut de recherche IMK proche de la Fondation Hans Böckler (le centre de recherche du syndicalisme allemand) et dirigé par Gustav Horn. Pour justifier un SMIC légal, I'IMK avait notamment publié en septembre 2008 une étude intitulée: "La France, un modèle pour l'Allemagne?" (IMK-Report, $\left.n^{\circ} 31 / 2008\right)$. Son résumé concluait: " La meilleure performance de la France révèle que la création d'emplois n'est pas obligatoirement liée à la modération salariale ». Car pour fonder la revendication d'un SMIC légal, il faut aussi faire appel à des « modèles » étrangers et recourir à des comparaisons internationales. L'instrument clé de cet organizing dans le contexte électoral étant «le fort accent mis sur la justice sociale et la dignité humaine » (Dörre, 2013), il faut, pour provoquer une large adhésion en Allemagne, y construire un « antimodèle » allemand et une situation « d'urgence sociale ». Et appeler en renfort l'opinion des partenaires européens de l'Allemagne, à commencer par la France, pour qu'au niveau politique, ils exercent une certaine pression sur le gouvernement fédéral sortant et à venir. En 2013, visiblement, ce mode d'action développé il y a plusieurs années déjà a gagné en ampleur et rayonne jusque dans l'ensemble de l'UE, porté par l'explosion du chômage dans les Etats sudeuropéens en difficulté et l'impératif « imposé » (et le refus obstiné) de mener des réformes structurelles pour rétablir leur compétitivité économique.

Cette "stratégie de démocratisation économique » (Dörre, 2013) poursuit une autre finalité encore : mettre à profit « la crise systémique du capitalisme financier » pour établir une "vision alternative de la société » (ibid.). En décembre 2013, le syndicat Ver.di avait adopté un manifeste, publié en trois langues (allemand, anglais et français), intitulé : « Donner un avenir à l'Europe sociale. Manifeste sur la politique européenne. Principes d'un modèle économique et social alternatif pour l'UE ». Après avoir posé que, dans l'UE, "la justice sociale [est] en recul ", analysé les "faiblesses fondamentales du modèle économique de l'Union européenne » parmi lesquelles une " coordination insuffisante de la po- 
litique salariale [qui] met en danger la zone euro ", il formule divers axes d'action. L'un d'entre eux préconise : "le mouvement syndical européen doit s'attaquer d'urgence à l'élaboration d'un concept de mise en place d'un salaire minimum assurant l'existence dans l'UE... La contribution impérative de l'Allemagne en faveur d'une politique de salaire minimum transnationale dans le cadre d'un modèle européen serait la mise en place d'un salaire minimum national légal... A l'échelle européenne, il faudrait définir des salaires minima... Les pays européens devraient tous envisager comme valeur cible une norme minimale nationale s'élevant à $60 \%$ du salaire moyen national, avec un objectif intermédiaire de $50 \%$ du salaire moyen national ".

Voilà pourquoi l'Allemagne est dépeinte, en interne et à l'extérieur, comme le pays de la précarité et de la pauvreté. C'est donc aussi pour mieux alimenter, via le slogan du "bon » ou " meilleur » travail, la revendication, formulée à l'échelon européen, d'une approche plus " solidaire » au sein de la zone Euro dans le cadre notamment du semestre européen. Si ce projet a partiellement échoué le 2 octobre 2013, la Commission s'étant mise d'accord pour ne retenir que la mise en place d'un « tableau de bord " statistique comprenant cinq " indicateurs sociaux " (taux de chômage, jeunes sans travail ni formation, revenu disponible des ménages, risque de pauvreté, inégalités de revenus), il reste vivace à l'approche des élections au Parlement européen - sous la forme par exemple de l'idée d'un "Pacte social européen » que préconisent certains parlementaires pour corriger les conséquences sociales de l'austérité et des réformes structurelles requises dans le cadre du semestre européen.

\section{La réalité allemande : la qualité de vie au travail ressentie}

Qu'en est-il réellement du travail de qualité ou de la qualité de vie au travail en Allemagne ? II n'y a pas de réponse simple et univoque à cette question pour au moins deux raisons. La première est qu'il n'existe pas de définition consensuelle sur ces notions, ni à l'échelon national, ni international. La deuxième est que, dès lors, les multiples sources statistiques utilisées sont partielles ou partiales, qu'elles sont établies selon des critères variables et qu'elles sont donc difficilement comparables entre elles. De plus, elles se classent en deux catégories, parfois entremêlées : les statistiques établies sous l'angle macro-économique ou de politique macro-économique qui opposent souvent travail de qualité et précarité ; et d'autre part les données subjectives établies sur la base de sondages menés auprès des salariés et qui mesurent principalement la qualité de vie au travail. L'Institut der deutschen Wirtschaft, proche du monde de l'industrie, les a passées en revue dans une étude détaillée (Schäfer et al., 2013).

Pourtant, une définition claire est incontournable pour en "déduire des principes directeurs » pour l'action du politique et du syndicalisme car elle permettrait de "rétablir l'équilibre des objectifs entre quantité et qualité » de l'emploi. Cette étude, publiée en septembre 2013, vient donc aussi en réponse à la thématisation de la question par le DGB et divers partis politiques (SPD, Verts, Die Linke).

L'Agenda pour le travail décent développé par l'OIT repose sur la Déclaration de l'OIT relative aux droits fondamentaux au travail de 1998 et " a pour objectif d'assurer que le progrès social accompagne le progrès de l'économie et du développement » (OIT). Elle suit 4 objectifs stratégiques : créer des emplois, garantir les droits au travail, étendre la protection sociale et promouvoir le dialogue social. L'OIT se contente de dresser des profils pays - mais n'a jamais fait d'évaluation de l'Allemagne. Les approches européennes, pour leur part (indicateurs de Laeken, travaux de la Commission), se basent principalement sur les statistiques officielles sur le marché de l'emploi et la protection sociale, et cherchent elles aussi à formuler des recommandations. Mais les données recueillies par pays sont difficilement comparables entre elles dans la mesure où, bien qu'elles cherchent une perspective élargie, elles ne tiennent pas compte
Pas de définition univoque du « travail de qualité »

Malgré leur quête d'objectivité, les statistiques sont partielles 
Les sondages identifient le ressenti mais ne permettent pas les comparaisons

Les contextes diffèrent trop

Les pièges linguistiques sont innombrables de tous les facteurs systémiques influant sur l'emploi ; elles ne prennent pas suffisamment en compte non plus les mutations du travail, se contentant de dresser un état des lieux à un instant « $T$ ». Ainsi, par exemple, la focalisation sur le segment des bas salaires entraîne automatiquement une lecture en termes de pénurie d'emplois de qualité, mesurés selon le niveau des salaires. Or « une forte proportion d'emplois hautement rémunérés et à durée indéterminée n'apporte rien à une société si une partie importante de la population n'a pas accès à l'emploi » (Schäfer et al., 2013). L'opposition entre travail 'normal' et précaire mène à des distorsions dans l'appréciation de la situation réelle, puisqu'elle ne peut prendre en considération le fait qu'un contrat de travail est un acte librement consenti par les deux parties et qu'il est le résultat d'un choix pour la solution la plus satisfaisante à la fois pour le salarié et l'employeur.

La mesure de la qualité du travail par sondages présente elle aussi quelques vices de conception. L'enquête européenne sur les conditions de travail de la Fondation européenne pour l'amélioration des conditions de vie et de travail (Eurofound) prend également pour critère d'évaluation de la qualité du travail le degré de satisfaction des salariés. Dans cette approche, l'Allemagne était bien classée, avec un taux de satisfaction élevé et stable depuis 1995 ; il frisait les $88 \%$ en 2009. Or en 2010 , les critères applicables ont été revus, afin de mieux cadrer avec la Stratégie Europe 2020. Depuis cette cinquième édition de l'enquête EWCS, ils incluent "les risques physiques, le temps de travail, la ségrégation fondée sur le sexe, l'équilibre entre vie professionnelle et vie privée, la représentation du personnel, l'organisation du travail, le stress au travail, le développement des compétences, les revenus, la santé et le bien-être " (www. eurofound.europa. eu).

Cette approche est problématique à plusieurs égards. Ainsi, les grandes différences dans les systèmes de protection sociale ou d'imposition des revenus rendent délicate la comparaison des revenus issus du travail : I'indicateur retenu est le revenu net mensuel, certes calculé en parités de pouvoir d'achat, mais il ne tient pas compte des prestations autres que le salaire comme les contributions patronales aux caisses de retraites d'entreprise ou professionnelles - or cet apport est important en Allemagne. En outre, elle ne permet guère d'évaluer à sa juste mesure la flexibilité du temps de travail puisque le jeu des critères retenus ne permet pas de pondérer la valeur réelle d'indicateurs comme la possibilité de quitter le lieu de travail pour une heure ou deux à des fins personnelles, le travail dominical ou les corridors de temps de travail. Quant à la dimension compétences et autonomie des décisions, elle place au même niveau la participation à une mesure de formation ou la possibilité de faire valoir ses propres idées dans le processus - or cette dernière surtout fait partie de la culture du travail en Allemagne et devrait donc, en tant qu'élément systémique, être pondérée différemment. Enfin, la dimension perspectives d'avenir exclut qu'un CDD (même un apprentissage !) puisse offrir des perspectives, puisqu'elle n'accorde pas la même valeur, par exemple, à la crainte subjective de perdre son emploi (elle croît avec l'âge et la durée de la présence dans l'entreprise) et au type de contrat de travail. Et pourtant, dans l'édition 2010 de I'EWCS, l'Allemagne se place bien pour la satisfaction au travail : 28,5\% des salariés se disent très satisfaits et $59,8 \%$ satisfaits (en France, respectivement $21,3 \%$ et $58,0 \%$ ). Les pas très satisfaits ou très insatisfaits sont $9,5 \%$ et $2,1 \%$ (en France : $17,0 \%$ et 3,6 \%). Or il n'en va pas de même des indicateurs sur la qualité du travail (salaire, qualité du temps de travail ou perspectives d'avenir), qui placent les deux pays à niveau à peu près équivalent.

Si les efforts réalisés pour tenter de mesurer la qualité du travail en Europe sont louables, l'exercice d'une mesure objective de la qualité du travail n'en reste pas moins délicat, voire impossible, tant les pièges sont nombreux. II en va ainsi de l'International Social Survey Programme (ISSP), dont l'une des questions par exemple porte sur la satisfaction au travail. Dans le questionnaire en langue allemande, la question est posée en ces termes : « quelle est la satisfaction que 
vous tirez en général de votre métier? ». Or ce terme de métier (Beruf) a une connotation totalement différente de celle d'emploi exercé (main job dans la version anglophone) ; on demandait donc en réalité aux Allemands s'ils étaient satisfaits de leur qualification professionnelle!

L'enquête de I'Initiative Neue Qualität der Arbeit (INQA), menée en 2004 en Allemagne, poursuivait le but de recueillir les appréciations des salariés sur leur situation personnelle et professionnelle afin d'en tirer des principes d'orientation pour définir un travail de qualité. Une telle approche est lourde de présupposés. Les critères retenus (notamment les plafonds de revenu mensuel, rapportés à une personne de surcroît) mènent en effet automatiquement à la classification des temps partiels dans la catégorie " mauvais travail » puisqu'ils ne répondent pas aux critères fixés. De même, la possibilité de se montrer créatif ou l'impression que le travail accompli " a du sens » ne sont pas des critères adéquats pour mesurer l'état des relations personnelles entre collègues ou avec le supérieur hiérarchique, puisqu'ils ne tiennent pas compte des liens de réciprocité un élément pourtant primordial pour évaluer les conditions de travail et leur qualité. Quant à l'index Gute-Arbeit du DGB, établi sur la base de sondages annuels et dans le prolongement de l'enquête INQA, il vise à déclencher un débat dans l'opinion. Les critères retenus sont donc guidés par la construction d'une image de "mauvais » travail (précarisation, paupérisation) pour légitimer une campagne en faveur d'un « meilleur » travail.

Toutes ces tentatives de définir la qualité du travail par des critères qui semblent à première vue objectifs et comparables (salaires, types de contrats, horaires...) tout en y incluant des éléments d'évaluation subjectifs ( $\mathrm{y}$ compris dans l'élaboration ou la pondération des indicateurs) montrent bien que l'exercice est impossible, tant sa complexité est grande puisque les éléments à prendre en considération pour avoir au moins une vision approximative de ce que pourrait recouvrir la notion de qualité du travail ou travail de qualité sont nombreux : ils incluent aussi bien des facteurs psycho-sociaux que les statistiques sur l'emploi, le mode de gouvernance des entreprises ou les systèmes de formation professionnelle, la structure des activités, etc. Et comment, dans ce contexte, trouver un compromis satisfaisant entre éléments subjectifs et objectifs - si jamais cela est possible ? La littérature sur ce sujet est abondante.

On peut donc se contenter de cet état de fait et travailler avec les données existantes - mais à une seule condition : qu'on en connaisse les limites. On peut tout autant se satisfaire plus modestement des nombreuses enquêtes cherchant à mesurer la satisfaction des salariés, donc la qualité de vie ressentie au travail - sachant que ces sondages ne permettent jamais que d'obtenir une photographie elle aussi partielle (et par nature partiale). Ne sont en effet pris en compte que les avis des salariés, jamais ceux de ceux qui les embauchent ; or la qualité du travail résulte de celle de leur coopération. Néanmoins, ces sondages peuvent donner un éclairage du vécu si on interprète les réponses obtenues à la lumière du cadre de vie national avec tous ses understatements.

On peut également se livrer à une lecture intelligente d'indicateurs comme ceux que fournit Destatis qui, dans son dernier rapport sur la question, recourt à une multitude de sources statistiques dont la plus importante est le Mikrozensus (Destatis, 2012). II retient sept grands indicateurs : sécurité et égalité hommes/ femmes, revenus et prestations d'entreprises, temps de travail et conciliation entre vies familiale et professionnelle, relations du travail, qualification et formation continue, coopération et motivation. On y apprend par exemple qu'en 2007, le stress (pénibilité psychique) touche surtout les milieux académiques (17,6\%), ainsi que les cadres intermédiaires et les dirigeants (16,9\%), les moins concernés étant les salariés dans le domaine des services et les vendeurs/vendeuses $(8,1 \%)$ comme les travailleurs auxiliaires $(5,0 \%)$. On constate que $1 \%$ seulement des actifs occupés n'ont aucune protection maladie en 2011 ; que les salariés à temps plein travaillent en moyenne 42 heures par semaine, mais que cette durée s'accroît avec l'âge et les responsabilités. On s'aperçoit que près de
Les enquêtes INQA et DGB présupposent un « mauvais travail »

Statistiques et sondages sont précieux, mais il faut les décrypter

\section{Destatis :}

$88 \%$ des Allemands satisfaits de leurs conditions de travail 
L'importance des facteurs culturels

La qualité du travail ressentie n'a pas varié en dix ans la moitié des actifs sont occupés depuis plus de dix ans dans l'entreprise où ils exercent en 2011 , et $20 \%$ depuis 5 à 10 ans ; un tiers seulement y travaille depuis moins de 5 ans, une proportion qui n'a guère varié au cours des 15 dernières années. Quant à la part des CDD, elle est inférieure en Allemagne à ce qu'elle est en France : 9,5\% des femmes et $8,6 \%$ des hommes ont un CDD outre-Rhin, de ce côté-ci, les taux sont respectivement de $11,9 \%$ et $10,2 \%$. Enfin, la part des bas salaires est inversement proportionnelle au niveau de qualification : $30 \%$ de ceux qui n'ont aucune qualification touchent un bas salaire, contre $4 \%$ seulement de ceux qui ont achevé des études supérieures dans les filières générales ou professionnelles. Si $84 \%$ des sondés affirment en 2010 que leur travail «a du sens », cette part varie avec le niveau de qualification ou la profession : le taux de réponses est de $96,5 \%$ chez ceux qui ont achevé des études supérieures, de $94,5 \%$ chez les agriculteurs et les pêcheurs, et de $92,2 \%$ chez les cadres et dirigeants; les travailleurs auxiliaires sont les moins convaincus (60,1\%). Au total, en 2010, $88 \%$ des Allemands se disent satisfaits de leurs conditions de travail (contre $78 \%$ des Français).

C'est aussi le mode de gouvernance des entreprises en Allemagne qui explique ce résultat, de même que le principe d'équité de droits entre capital et travail qui s'exprime entre autres dans la co-responsabilité salariés/patron. De même, il faut prendre en considération le fait que c'est l'entreprise - surtout les PME du Mittelstand - qui socialise près de la moitié des Allemands grâce à l'apprentissage. Les salariés allemands s'identifient donc beaucoup plus facilement que d'autres à leur entreprise, ce qui se répercute sur leur motivation. C'est ce type de soft skills qui composent la grille de lecture des sondages. Comment les comparer à l'échelle européenne sinon par un travail approfondi et de longue haleine pour faire apparaître tous ces facteurs culturels qui forgent les identités et déterminent la valeur travail ?

Quand on prend pour référence les multiples études sur la satisfaction des Allemands au travail, le tableau est très différent de l'image catastrophiste propagée dans l'espace public. Depuis le milieu des années 1990, «la qualité du travail, telle qu'elle est ressentie par les salariés, est ... restée constante malgré l'extension du secteur des bas salaires et la hausse des formes d'emploi qui ... se distinguent de l'emploi dit normal ॥ conclut ainsi une étude consacrée à une lecture fine de l'enquête EWCS 2010 (Hammermann/Stettes, 2013). Elle révèle par exemple qu'un Allemand sur deux (48\%) se dit satisfait de son salaire et que, parmi les autres, $82 \%$ (nettement plus que les Européens du Sud) disent que leur travail les satisfait; c'est là le reflet d'un marché du travail somme toute plus inclusif dans le nord de l'Europe. Pour ce qui est du stress ou des horaires atypiques, les Allemands affirment certes un peu plus souvent que les autres Européens avoir de fortes contraintes de temps, subir des cadences infernales ou se sentir stressés, mais cela influe visiblement peu sur leur satisfaction au travail, puisque $85 \%$ d'entre eux (6,5 points de plus que la moyenne de I'UE 27) se disent satisfaits de leur travail. C'est que, en Allemagne, la sécurité et la santé au travail sont depuis longtemps au cœur de l'organisation des process de production, et que donc les facteurs stressants comme le bruit ou les températures extrêmes sont moins prononcés que dans d'autres pays. S'ajoute à cela une gestion participative des ressources humaines qui réduit elle aussi le stress psychique. Enfin, les fonctions occupées en adéquation avec la qualification jouent elles aussi en faveur de ce bon climat dont le 'secret' réside non pas tant dans des indicateurs économiques que dans des facteurs systémiques comme l'autonomie individuelle et le respect du collaborateur, considéré dans sa qualité de personne-ressource.

« UN TRAVAIL EST BON QUAND IL REFLĖTE LES ATTENTES DE CELUI QUI L'OCCUPE ». C'est en ces termes que la Confédération syndicale DGB définit la qualité du travail sur son site dédié à l'Index Gute-Arbeit, après avoir posé : " Est social ce qui crée du bon travail » (www.dgb-index-gute-arbeit.de). Une définition circu- 
laire et aussi séduisante car offrant tant de surfaces de projection ne peut que susciter une vaste adhésion dans l'opinion et fédérer une infinie variété de perspectives dans un objectif de " plus de justice sociale ", tel qu'il figure en tête de l'agenda électoral - des élections au Bundestag le 22 septembre dernier aux européennes au printemps prochain, en Allemagne comme ailleurs.

Si cet objectif construit sur le concept de travail de qualité a pu ainsi se propager outre-Rhin, c'est aussi qu'il reflète une tendance de fond dans l'opinion allemande depuis la fin des années 1990. Déjà, lors de la campagne électorale de 1998, le leitmotiv de l'opposition au gouvernement Kohl sortant portait sur un déséquilibre en matière de justice sociale. En février 2013, $63 \%$ des Allemands restent persuadés que les revenus et le patrimoine sont injustement répartis (IfD Allensbach, 2013). Voilà qui ouvre un boulevard à la construction de l'image d'une Allemagne " anti-sociale »...

Or ce sondage révèle aussi que, si les Allemands sont très critiques dès qu'on leur demande leur avis sur leur propre pays, considérant tout ce qui est perfectible, ils se montrent au contraire très attachés et confiants dans leur modèle social quand il s'agit de comparaisons internationales. Dans ce cas, l'Allemagne vient en deuxième position ( $23 \%$ de réponses à « la plus grande justice sociale ») après la Suède (34\%), la France ne recueillant que $3 \%$ d'avis positifs. Et quand on entre plus avant dans ce qu'ils entendent par justice sociale, on s'aperçoit qu'ils placent certes en tête le fait de pouvoir vivre de son salaire (91\%), mais que peu après ils affirment que "celui qui travaille plus et mieux doit aussi gagner plus que celui qui en fait moins " (70\%). Autrement dit, ce qui leur importe, ce n'est pas une approche égalitariste telle que la véhicule la revendication d'un SMIC légal généralisé, mais bien plutôt celle d'une solidarité fondée sur l'équité des chances. Leurs priorités sont alors la garantie des retraites, la baisse du chômage, ou encore la stabilité des prix. Et ils estiment (à $65 \%$ ) que c'est le politique qui est le mieux à même de garantir cette justice sociale comprise comme équité des chances, les entreprises ne recueillant que $32 \%$ des réponses, et les syndicats le moins (21\%).

Le débat sur le travail de qualité 'surfe' sur de telles attentes. Pour les entretenir dans l'opinion, le mouvement syndical mise sur toutes les forces établies et alternatives. Alors qu'il était le défenseur des intérêts de tous les salariés et en cette qualité un acteur majeur et rassembleur dans le jeu des institutions, le syndicalisme allemand tend aujourd'hui à se 'politiser' et à développer une fonction tribunicienne pour construire un autre type d'identification. II 's'européanise' aussi, cherchant par-delà les frontières des alliés pour défendre une cause où les intérêts des salariés sont aujourd'hui secondaires. La priorité est de mobiliser une base militante sur la vision d'une "Europe sociale » qui corrigerait les " errements " d'une union monétaire forçant austérité et démantèlement des acquis sociaux puisqu'elle ne permet plus de dévaluer la monnaie pour combler les écarts de compétitivité. Autour du thème du travail de qualité, on se livre en Allemagne et ailleurs - à une bataille de chiffres visant à prouver une misère qui n'existe pas, du moins dans ces proportions, et dont le semblant de scientificité ou d'objectivité masque l'impossibilité de définir l'objet du débat et vise à entretenir une connivence implicite entre discutants du même bord. Et pourtant, contrairement aux apparences ainsi cultivées, les Allemands ne sont pas plus mal lotis que les Français. Ils semblent même globalement plus épanouis au travail, ce dont atteste leur capacité à innover en permanence et à assurer la compétitivité de leur entreprise comme du site de production Allemagne.

\section{Indications bibliographiques}

- Balz A., KRell Noll H.-H., Weick S., « Unsicherheit des Arbeitsplatzes mindert Arbeitszufriedenheit besonders in Deutschland. Vergleichende Analysen zur Wahrnehmung und Bewertung der Arbeitsbedingungen in Europa »,GESI, ISI, $n^{\circ}$ 48/2012

- BISPINCK R., SCHULTEN T., "Re-Stabilisierung des deutschen Flächentarifvertragssystems », IAQStandpunkt, $\mathrm{n}^{\circ}$ 2/2012 
- Bohulskyy Y., ERLinghagen M., Scheller F., « Arbeitszufriedenheit in Deutschland sinkt langfristig. Auch geringe Arbeitszufriedenheit im europäischen Vergleich », IAQ-Report, n 3/2011

- BONSE E., « Agendapolitik durch die Hintertür », Mitbestimmung, n 4/2013

- Bosch G., « Prekäre Beschäftigung und Neuordnung am Arbeitsmarkt. Expertise im Auftrag der Industriegewerkschaft Metall. Duisburg, September 2012 », IAQ-Standpunkt, n²/2012

- Bosch G., « Prekäre Beschäftigung und Neuordnung am Arbeitsmarkt. Statement auf der Pressekonferenz der IG Metal »", Berlin, 28 septembre 2012

- Bosch G., "La progression des bas salaires en RFA plaide pour un salaire minimum légal », Regards sur l'économie allemande, n 93/2009

- BouRgeoIs I., «Intérim : un SMIC légal, faute de représentativité syndicale ? », Regards sur l'économie allemande, $\mathrm{n}^{\circ} 86 / 2008$

- Bourgeols I., « Marché postal allemand : libéralisation sans concurrence », Regards sur l'économie allemande, $\mathrm{n}^{\circ} 85 / 2008$

- Bourgeols I., «Directive 'Services' : les enjeux du débat allemand », Regards sur l'économie allemande, $\mathrm{n}^{\circ} 71 / 2005$

- BRENKE K., "Vrais et faux enjeux de la controverse sur les salaires minima légaux en RFA », Regards sur l'économie allemande, $n^{\circ}$ 94/2009

- BRENKE K., MÜLLER K.-U., « Gesetzlicher Mindestlohn - Kein verteilungspolitisches Allheilmittel », DIW Wochenbericht, $\mathrm{n}^{\circ}$ 39/2013

- BRINKmanN U., NAChtwey O., "Krise und strategische Neuorientierung der Gewerkschaften », Aus Politik und Zeitgeschichte, Dossier „Gewerkschaften“, n 13-14/2010

- BUNDESMINISTERIUM FÜR ARBeIt UNd SOZIALES, Initiative Neue Qualität der Arbeit, www.bmas.de/ DE/Themen/Arbeitsschutz/Projekte-und-Initiativen/INQA/initiative-neue-qualitaet-der-arbeit.html

- DöRRE K., « Organizing - ein Konzept zur Erneuerung gewerkschaftlicher Organisationsmacht », Dossier „Internationale Solidarität Revisited“, septembre 2013, www.migration-boell.de/web/ integration/47_3806.asp

- DörRE K., « Gewerkschaftliche Erneuerung: Aus der Krise zu neuer Stärke? », Emanzipation, $n^{\circ} 2 / 2012$

- DöRRE K., « Die strategische Wahl der Gewerkschaften - Erneuerung durch Organizing ? », WSI Mitteilungen, $\mathrm{n}^{\circ} 1 / 2008$

- Hammermann A., Stettes O., «Qualität der Arbeit - zum Einfluss der Arbeitsplatzmerkmale auf die Arbeitszufriedenheit im europäischen Vergleich », IW-Trends, $n^{\circ} 2 / 2013$

- Institut für Demoskopie Allensbach, Was ist gerecht? Gerechtigkeitsbegriff- und Wahrnehmung der Bürger, février 2013

- Kalina T., WEINKOPF C., « Niedriglohnbeschäftigung 2011. Weiterhin arbeitet fast ein Viertel der Beschäftigten in Deutschland für einen Niedriglohn ", IAQ-Report, n 1/2013

- Kalmbach P., SchumanN M., « Finanzkrise als Schocktherapie », WSI-Mitteilungen, n 11+12/ 2008

- LASSERRE R., « Nouveaux enjeux de la politique salariale », Regards sur l'économie allemande, $\mathrm{n}^{\circ} 75 / 2006$

- LATTARD A., « Négociation collective : quel avenir pour la convention de branche ? », in BouRGEOIS I. (dir.), Le modèle social allemand en mutation, Editions du CIRAC, 2005

- Noll H.-H., WEICK S., « Nicht einmal jeder Dritte empfindet soziale Unterschiede in Deutschland als Gerecht », GESI, ISI, n 48/2012

- OIT, Rapport mondial sur les salaires 2012/13. Salaires et croissance équitable, Genève, 2013

- PICKshaus K., « Gute Arbeit - Schönwetterthema oder Element offensiver Krisenbewältigung ? », Sozialismus, $n^{\circ} 4 / 2009$

- RHEIN T., « Erwerbseinkommen. Deutsche Geringverdiener im europäischen Vergleich », IABKurzbericht, $n^{\circ} 15 / 2013$

- SChÄFer H., Schmidt J., Stettes O., « Qualität der Arbeit », IW Positionen, n 61, 2013

- SCHROEDER W., « Eigene Stärke braucht Kooperationspartner », Dossier „Debatte Krisenkorporatismus“, Mitbestimmung, $n^{\circ}$ 4/2013

- SCHROEDER W., « ,Le modèle syndical allemand n'existe plus' (un entretien) », et « IG metall pour un Etat social plus équitable », in Bourgeols I. (dir.), Le modèle social allemand en mutation, Editions du CIRAC, 2005

- Sommer M., « Konturen einer Neuen Ordnung der Arbeit », in HANS BöckLeR STIFTUNG, WSI, DGB, Tagungsdokumentation „Konturen einer Neuen Ordnung der Arbeit", Berlin, 29 mai 2013

- SpItznagel E., « Un marché de l'emploi en mutation », Regards sur l'économie allemande, n 91/ 2009

- Statistisches Bundesamt, « Geld verdienen und was sonst noch zählt. Qualität der Arbeit, $2012 »$, www.destatis.de

-WAHL S., "Les vraies causes de la disparité des revenus en RFA », Regards sur l'économie allemande, $\mathrm{n}^{\circ} 88 / 2008$

- ZIMmERMANN K. F., « Reflektionen zur Zukunft der Arbeit », IZA Standpunkte, n 56/2013. 